\title{
Analysis of electrode microbial communities in an up-flow bioelectrochemical system treating azo dye wastewater
}

\author{
Min-Hua Cui ${ }^{a}$, Dan Cui ${ }^{b}$, Lei Gao ${ }^{a}$, Hao-Yi Cheng ${ }^{b}$, Ai-Jie Wang, \\ a State Key Laboratory of Urban Water Resource and Environment, Harbin Institute of Technology, Harbin 150090, PR China \\ ${ }^{\mathrm{b}}$ Key Laboratory of Environmental Biotechnology, Research Center for Eco-Environmental Sciences, Chinese Academy of Sciences, Beijing 100085, PR China
}

\section{A R T I C L E I N F O}

\section{Article history:}

Received 20 July 2016

Received in revised form 17 October 2016

Accepted 18 October 2016

Available online 18 October 2016

\section{Keywords:}

Bioelectrochemical system (BES)

Microbial community structure

Azo dye

Electrode polarity and position

\begin{abstract}
A B S T R A C T
Bioelectrochemical system (BES) is a rapidly developing technology covering contamination remediation, resource recovery and power generation. Electrode biofilms play a key role in BES operation. In this work, a single chamber up-flow bioelectrochemical system (UBES) was assembled with two preinoculated anodes and two raw cathodes for azo dye wastewater treatment. Microbial community structures of these electrodes after long-term operation (more than 200 days) were carried out by highthroughput Illumina 16S rRNA gene MiSeq sequencing platform. Microorganisms belonging to Enterobacter, Desulfovibrio and Enterococcus, which are capable of bidirectional extracellular electron transfer, were found to be the dominant members in all biofilms. Neither the polarity nor the position of the electrodes obviously altered the microbial community structures. This study provides a feasible strategy to build electrode active biofilms in a BES for azo dye wastewater treatment and gives great inspirations to bring this technology closer to application.
\end{abstract}

(c) 2016 Published by Elsevier Ltd.

\section{Introduction}

Electrochemical technology demonstrates tremendous potential for water and wastewater treatment in recent years, due to the outstanding specialties such as high efficiency, complete mineralization, extensive applicability and relative easy to manipulate [1,2]. However, high cost and sustaining energy consuming seem to be the barrier for implementing this technology in the practical pollution remediation, especially considering the potential application in large scale wastewater treatment plant. By now, biological treatment is still the most widely applied wastewater remediation method. But the pure biological facilities can no longer satisfy the increasing wastewater amount and the stricter wastewater discharging standard [3-5]. It is urgent to explore a feasible, sustainable and economical strategy to deal with the wastewater issue.

Bioelectrochemical system (BES) is a newly developed technology which integrates advantages both from conventional electrochemical and biological processes. With a small voltage supplied, BESs are capable of azo bond cleavage [6], nitro reduction [7],

\footnotetext{
* Corresponding author at: State Key Laboratory of Urban Water Resource and Environment, Harbin Institute of Technology (SKLUWRE, HIT), P.O. Box 2614, 202 Haihe Road, Harbin, 150090, PR China.

E-mail address: waj0578@hit.edu.cn (A.-J. Wang).
}

heavy metal recovery [8], sulfur transformation [9] and dehalogenation [10]. In some cases, BESs are used for recovering energy resources from waste(water)s in the form of $\mathrm{H}_{2}$ [11], $\mathrm{CH}_{4}$ [12] and electricity $[13,14]$. BES has been proved the superiority such as excellent performance and time-saving comparing to conventional anaerobic processes [15], and more cost-efficient and environment-friendly versus electric or electrochemical methods due to lower energy supplied and less by-products $[7,16]$. BES seems to be an alternative approach to deal with the environment pollution as well as the energy shortage, though it still need to be burnished in view of practical application.

The feasibility of BES has been verified by plenty of studies, however, the microbial analysis of electrode biofilm is lagging behind. Biofilm is the fundamental component in a BES. Organic matter is oxidized in bioanode to release electrons by anode respiration bacteria, then flow to cathode to drive the reductive reaction directly or indirectly mediating by cathode biofilm [17]. Anode microbial consortia play a key role in BES and would be potentially affected by plenty of factors, such as resistance in the external circuit, co-substrate type (electron donor source), potential setting, with or without separator and $\mathrm{pH}$ [18], especially the inoculum source. Lin et al. investigated the effect of inocula that from river sediment, activated sludge and anaerobic sludge on microbial fuel cells (MFCs) performance. Anaerobic sludgeinoculated one presented rapid biofilm development and higher 
substrate utilization, while river sediment-inoculated one achieved the highest power output [19]. However, different result was reported by Gao et. al., MFC was rapid start-up and had better electricity generation performance inoculating with aerobic activated sludge than anaerobic sludge [20]. A lot of previous studies have demonstrated that BESs equipped with biocathode would increase reaction kinetics by biocatalysis and decrease the energy consumption by overpotential reduction $[10,16]$. Butler et al. used a denitrifying biocathode to reduce perchlorate and achieved a maximum removal rate of $24 \mathrm{mg} /(\mathrm{Ld})$ with cathodic conversion efficiency of 84\% [21]. Gregory and Lovley reported uranium (U) could be rapidly removed from solution by an abiotic cathode yet $\mathrm{U}(\mathrm{VI})$ returned to solution after applied voltage removed. With a G. sulfurreducens adhered to cathode, reduced $U$ (VI) was stably precipitated [22].

To explore an efficient strategy to establish electrode active biofilms is crucial for successfully operating a BES. The feasibility and superiority of the up-flow bioelectrochemical system (UBES) for treating azo dye wastewater have been verified in previous studies $[6,23]$. However, little is known about the effect of up flow pattern, azo dye as well as continuous electric field on electrode microbial community structures. In order to gain insight into these interactions influencing on the microbial communities in the longterm operating UBES (more than 200 days), 16S rRNA gene based high-throughput Illumina MiSeq sequencing platform was employed to analyze biofilms from electrodes in different positions and polarities.

\section{Materials and method}

\subsection{Reactor configuration and operation}

The UBES was manufactured of plexiglass with working volume of 1.5 L [24]. Graphite fiber brushes were used for both anode and cathode, and each electrode consisted of 8 small graphite fiber brushes $(2.5 \mathrm{~cm}$ in diameter and $4 \mathrm{~cm}$ in length, graphite fiber produced by TOHO TENAX, Co., Ltd., Japan). Two pairs of electrodes were installed into the reactor, arranged as anode, cathode, anode, cathode, from top to the bottom.

Before assembling the UBES, the anodes were preinoculated in a dual-chamber BES at a fixed potential of $-400 \mathrm{mV}$ vs. a saturated calomel electrode $(+247 \mathrm{mV}$ vs. standard hydrogen electrode) controlled by a potentiostat (WMPG1000K8, Wontech International Co., Ltd., Korea). The BES operated in batch mode and the medium composition used in this stage was glucose $(2000 \mathrm{mg} / \mathrm{L})$, phosphate buffer solution (PBS, $50 \mathrm{mM}, \mathrm{pH} \sim 7.2), \mathrm{KCl}(130 \mathrm{mg} / \mathrm{L})$, $\mathrm{NH}_{4} \mathrm{Cl}(310 \mathrm{mg} / \mathrm{L})$, trace element solution $(10 \mathrm{~mL} / \mathrm{L})$ and vitamin solution $(10 \mathrm{~mL} / \mathrm{L})$ [25]. In the first two cycles, $50 \mathrm{~mL}$ anaerobic activated sludge (obtained from Taiping Municipal Wastewater Treatment Plant, Harbin, China; SS of $31 \mathrm{~g} / \mathrm{L}$ and VSS of $15 \mathrm{~g} / \mathrm{L}$ ) was added into the reactor as seed sludge. When a stable current output was observed, the anodes were considered to be successfully inoculated. After that, these inoculated anodes and the raw cathodes were assembled in UBES.

During the experiment, each pair of electrodes was operated with external resistance of $10 \Omega$ and applied voltage of $0.5 \mathrm{~V}$ using a DC power supply (FDPS-180, Fudan Tianxin Scientific and Educational Instruments Co., Ltd, Shanghai, China). According to previous work [26], $0.5 \mathrm{~V}$ applied voltage was sufficient and economical for AYR decolorization. A saturated calomel electrode was used as reference electrode. Anodes, cathodes and reference electrode were connected to a data acquisition system (Keithley 2700, Keithley Co. Ltd., U.S.), currents were recorded at every $10 \mathrm{~min}$.

The composition of medium was glucose $(500 \mathrm{mg} / \mathrm{L})$, Alizarin yellow R (AYR, 50-200 mg/L), PBS (50 mM, pH 7.2), KCl (130 mg/
L), $\mathrm{NH}_{4} \mathrm{Cl}(310 \mathrm{mg} / \mathrm{L})$, trace element solution $(10 \mathrm{~mL} / \mathrm{L})$ and vitamin solution $(10 \mathrm{~mL} / \mathrm{L})$. The influent AYR loading rates gradually increased $\left(100,200,400,600,800 \mathrm{~g} \mathrm{AYR} / \mathrm{m}^{3} \cdot \mathrm{d}\right)$ by adjusting the influent AYR concentration and hydraulic retention time (HRT).

\subsection{Chemicals and analytical method}

AYR that contains an azo bond and a nitro group was used as the model azo dye (commercial purity grade, Shanghai Sangon Biotech Co., Ltd., China). AYR concentration was quantified by a UV-vis spectrophotometer (UV-1800, Shanghai Meipuda instrument Co., Ltd., China) at a wavelength of $374 \mathrm{~nm}$.

\subsection{Biofilms sampling and DNA extraction}

Biofilms were sampled for DNA extraction. Graphite fibers were cut from anodes and cathodes, respectively, and fragmented using sterile scissors. Biofilm samples taken from at least three different small brushes were combined together for DNA extraction. Each sample was put into a $1.5 \mathrm{~mL}$ sterile centrifuge tube with $1 \mathrm{~mL}$ sterile deionized water, which was then mixed by a shaker flowed by centrifuging at $13,000 \mathrm{rpm}$ for $10 \mathrm{~min}$ with removing the supernatants. This procedure was repeated twice for maximizing DNA extraction from biomass samples. Collected pellets were stored in $2 \mathrm{~mL}$ sterile centrifuge tubes at $-70^{\circ} \mathrm{C}$ before DNA extraction. DNA extraction as well as 16S rRNA gene based Illumina MiSeq sequencing of the pellets was served by Shanghai Sangon Biotech Co., Ltd.

\subsection{High-throughput $16 S$ rRNA gene Illumina MiSeq sequencing}

Amplicon libraries were constructed by Illumina Miseq PE300 using bacterial Nobar 341F (CCTACGGGNGGCWGCAG) and Nobar 805R (GACTACHVGGGTATCTAATCC). Both forward and reverse primers were added with barcode. PCR amplification, products purification and quantification, and the sequencing were carried out through the Illumina MiSeq platform in Shanghai Sangon Biotech Co., Ltd.

\section{Results and discussion}

\subsection{Decolorization in the up-flow bioelectrochemical system}

The up-flow bioelectrochemical system (UBES) was employed to decolorize AYR. The highest AYR decolorization efficiency of $87.74 \pm 3.52 \%$ emerged at hydraulic retention time (HRT) of $12 \mathrm{~h}$ with influent AYR concentration of $200 \mathrm{mg} / \mathrm{L}$. Since the HRT declined to $6 \mathrm{~h}$ corresponding to the highest AYR loading rate of $800 \mathrm{~g} /\left(\mathrm{m}^{3} \cdot \mathrm{d}\right)$, AYR decolorization rate reached maximum of $524.43 \pm 25.07 \mathrm{~g} /\left(\mathrm{m}^{3} \cdot \mathrm{d}\right)$. Total current of the UBES was $17.86 \mathrm{~mA}$. If all electrons through external circuit was assumed for driving AYR decolorization, this current was equal to $76.51 \mathrm{mg}-\mathrm{AYR} / \mathrm{L}$ which given a contribution of $59.51 \%$ for gross AYR decolorization. It indicated that more than half of the AYR reduction was relative to the electrochemical process. When the applied voltage was removed and the UBES operated at open circuit with AYR loading rate kept at $800 \mathrm{~g} /\left(\mathrm{m}^{3} \cdot \mathrm{d}\right)$, decolorization efficiency significantly decreased to $57.58 \pm 6.15 \%$. These results clearly demonstrated the superior performance in AYR decolorization and emphasized the significant contribution of electrochemical reduction [24].

\subsection{Overall microbial community structures}

Illumina sequencing platform was employed to analyze the microbial communities from four electrodes of the UBES. The rarefaction curves at 3\% distance thresholds was shown in Fig. 1. 


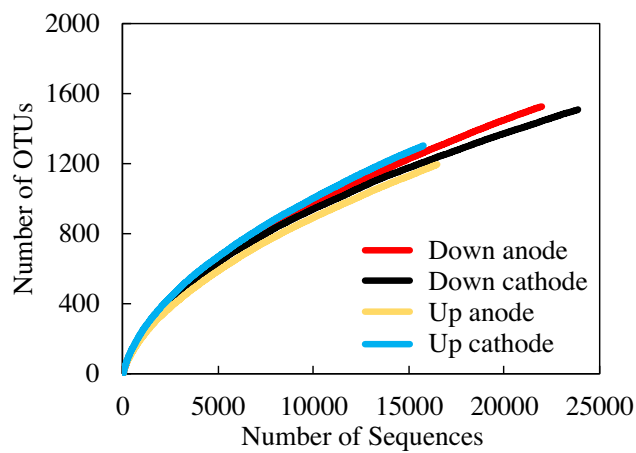

Fig. 1. Rarefaction curves of microbial communities from four samples (at $3 \%$ distance thresholds).

Over 15000 high quality sequences $(22030,23885,16503$ and 15801 for down anode, down cathode, up anode and up cathode, respectively) were obtained from each sample. Relative more operational taxonomic units (OTUs) were observed in samples from down electrodes ( 1522 for anode and 1505 for cathode) than that of the electrodes at upside (1197 for anode and 1304 for cathode). Obviously, none of the rarefaction curves reached a plateau, and new OTUs continued to emerge even after 15000 sequences. It indicated that Illumina sequencing could reveal the higher diversity of microbial communities in UBES. The estimated species richness abundance-based coverage estimator (ACE) and Chao1 were also consistent with this trend, as shown in Table 1. Based on the Shannon index, the up cathode (4.31) had relative higher diversity than that of the corresponding anode (4.00) and the down electrodes ( 4.05 for both anode and cathode). It was notable that the up cathode community had relative higher biodiversity and unique OTUs in the same reactor but different position. All the Coverage value among four samples varied from 0.95 to 0.97 indicating that the sequencing results were reliable to reflect the characteristics of the microbial communities.

Principal component analysis (PCA) indicated that two anode samples clustered together and presented high similarity of the microbial communities (Fig. 2). It is reasonable considering both the up and down anodes were preinoculated before assembling in the UBES and shared the same inoculum. Two cathodes located far from two anodes and separated from each other in PCA, it was probably attributed to the raw status of cathodes before installing in the reactor. The total classified OTUs in four samples was 3095 and 460 OTUs (14.86\%) were shared by them. Thereinto, Proteobacteria (33.46\%), Firmicutes (28.02\%) and Bacteroidetes (17.51\%) were enriched at phylum level. Actinobacteria (4.28\%), Synergistetes (3.89\%) and Chloroflexi $(3.11 \%)$ were one order of magnitude lower in relative abundance (Fig. 3 ).

\subsection{Microbial community structures at phylum and class levels}

A total of 26 phyla were identified, $21,23,23$ and 22 for the down anode, down cathode, up anode and up cathode, respectively. As shown in Fig. 4A, the general structures were quite similar in four microbial communities. Proteobacteria dominated in all

Table 1

Summary of sequencing data for four samples (at 3\% distance thresholds).

\begin{tabular}{lllll}
\hline & ACE & Chao 1 & Shannon & Coverage \\
\hline Up anode & 4342 & 2659 & 4.00 & 0.96 \\
Up cathode & 4271 & 2876 & 4.31 & 0.95 \\
Down anode & 4933 & 3444 & 4.05 & 0.96 \\
Down cathode & 4546 & 3131 & 4.05 & 0.97 \\
\hline
\end{tabular}

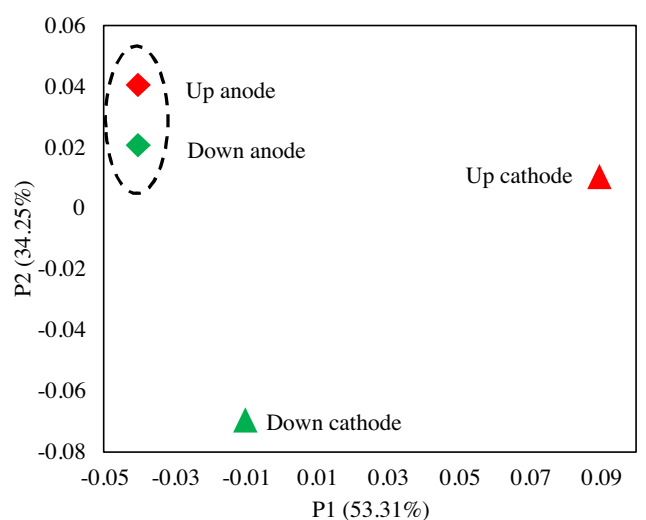

Fig. 2. Principal component analysis (PCA) of microbial communities from four samples based on the classified OTUs.

samples and accounted for approximate half of the relative abundance of total identified phyla, which was also found to be dominated (39.8\%) in a microbial community from an upflow anaerobic sludge blanket and BES coupling reactor for nitro reduction and dechlorination of 2,4-dinitrochlorobenzene [27]. The relative abundances of Firmicutes in two anodes microbial communities ( $19.22 \%$ in down anode and $19.87 \%$ in up anode) were quite similar, 15.90 and $22.89 \%$ in down and up cathode, separately. Firmicutes, obligate or facultative aerobes, were found to be a major component in MFC [28]. Besides, Firmicutes were sensitive to the electron donor types and enriched with glucose and propionate feeding condition $[29,30]$. Bacteroidetes had relative abundances from 11.51 to $14.72 \%$ in all samples and were often found in many BESs [28]. The relative abundances of Actinobacteria were 10.25, $7.45,9.24$ and $6.83 \%$ in down anode, down cathode, up anode and up cathode, respectively.

A total of 38 classes were classified and the most of the sequences were identified from 7 classes (Fig. 4B). Gammaproteobacteria which belonged to Proteobacteria was absolutely dominated in all four samples, indicating the similar microbial community structures among four electrode biofilms. Deltaproteobacteria enriched to $10.53 \%$ (anode) and $8.76 \%$ (cathode) in the down electrodes which slightly decreased to $8.15 \%$ in up anode and $6.38 \%$ in up cathode. Bacilli was the most enriched class belonged to Firmicutes and followed by Clostridia and Negativicutes. Besides, classes of Bacteroidia and Actinobacteria accounted for the relative high abundance in phyla of Bacteroidetes and Actinobacteria, respectively.

\subsection{Potential function of dominant genera}

A more in depth characterization of the microbial community was performed at a genus level. A total of 329 genera were classified from the tested samples. 18 genera with relative abundance $>1 \%$ in the four microbial communities were shown in Table 2. Enterobacter obviously dominated in all samples (relative abundance varied from 30.61 to $40.06 \%$ ) which was capable of azo dye reduction [31] as well as electricity generation [32]. Desulfovibrio presented $>5 \%$ of relative abundance in all samples and was reported to be involved in electricity generation [33] and azo dye reduction [34]. Enterococcus was enriched in both anodes (5.93\% for down and 5.19\% for up) and decreased in the cathodes (2.55 and $2.88 \%$ for down and up, respectively). Enterococcus was reported as a functional genus for azo dye and nitro-compounds reduction by previous literatures [35-37], and also dominated in a nitroaromatics nitrobenzene-reducing biocathode BES [38-40]. The relative abundances of Klebsiella among 


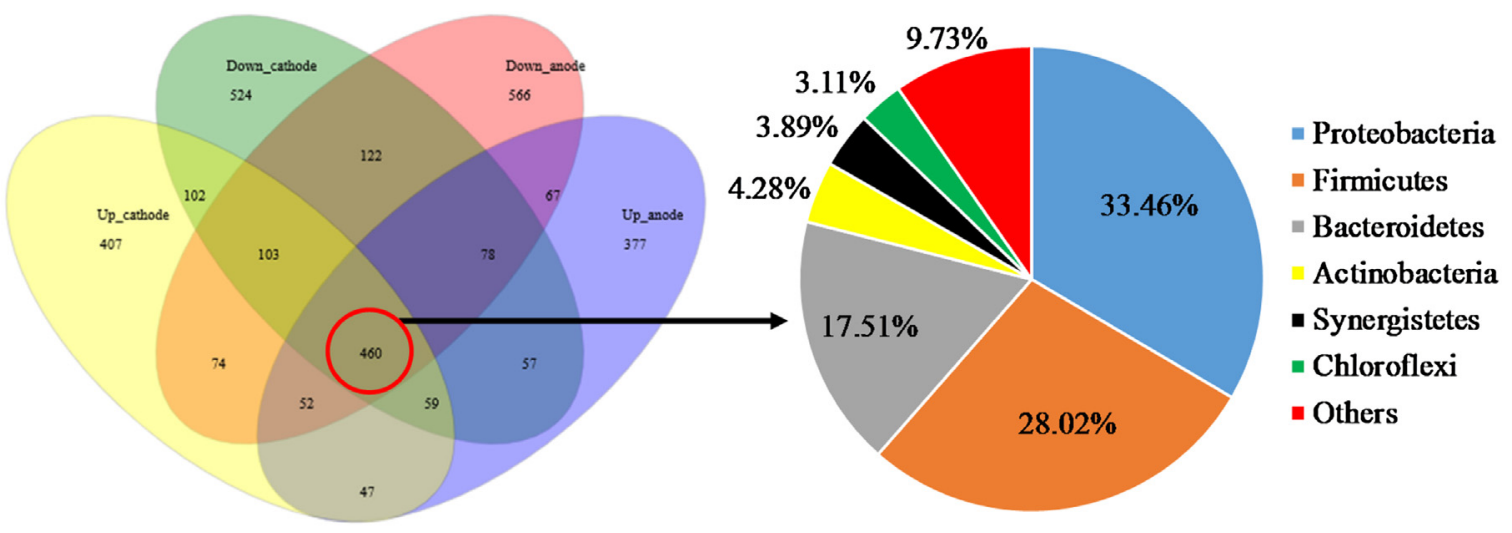

Fig. 3. Overlap of microbial communities from four samples. The shared OTUs were analyzed at phylum levels.

A
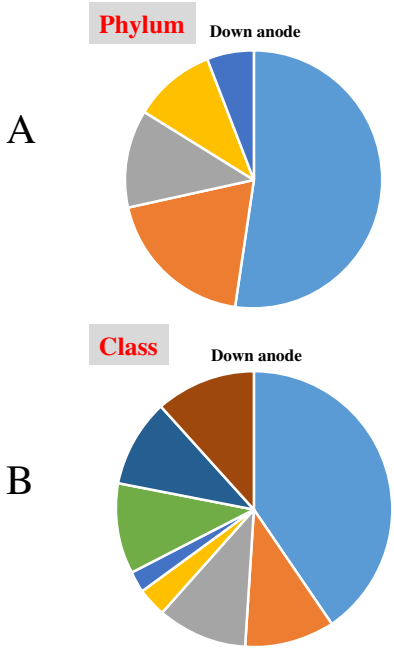
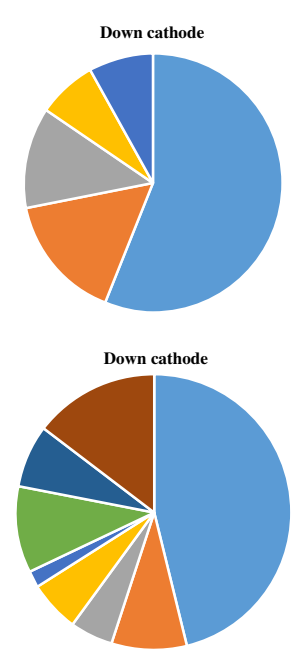
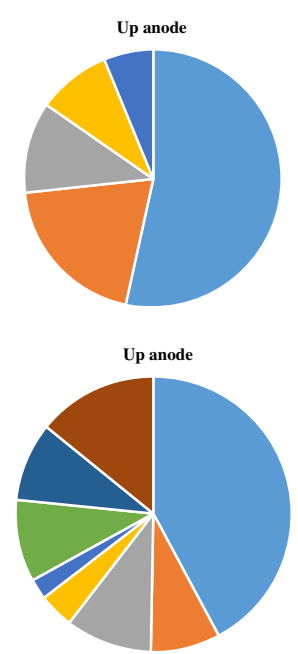

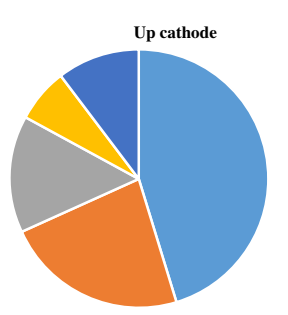

- Proteobacteria

- Firmicutes

- Bacteroidetes

- Actinobacteria - Others

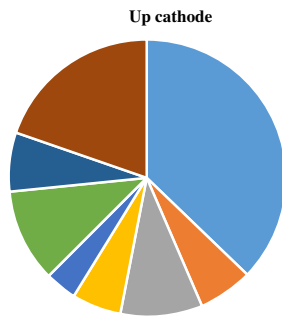

Proteobacteria Gammaproteobacteria Deltaproteobacteria Bacilli Firmicutes Clostridia

- Negativicutes

- Bacteroidia

- Actinobacteria

- Others

Fig. 4. Taxonomic classification of $16 \mathrm{~S}$ rRNA gene sequences from four microbial communities at the phylum and class levels.

Table 2

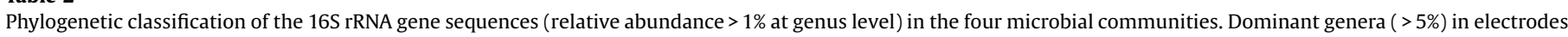
were bolded.

\begin{tabular}{|c|c|c|c|c|c|c|c|}
\hline Phylum & Class & Family & Genus (\%) & Down anode & Down cathode & Up anode & Up cathode \\
\hline Proteobacteria & Gammaproteobacteria & Enterobacteriaceae & Enterobacter & 34.07 & 40.06 & 33.29 & 30.61 \\
\hline Proteobacteria & Gammaproteobacteria & Enterobacteriaceae & Klebsiella & 3.18 & 3.98 & 2.79 & 3.83 \\
\hline Proteobacteria & Gammaproteobacteria & Enterobacteriaceae & Citrobacter & 2.11 & 0.95 & 1.84 & 1.01 \\
\hline Proteobacteria & Gammaproteobacteria & Moraxellaceae & Acinetobacter & 0.31 & 0.29 & 3.50 & 0.54 \\
\hline Proteobacteria & Deltaproteobacteria & Desulfovibrionaceae & Desulfovibrio & 9.62 & 8.16 & 7.45 & 5.21 \\
\hline Proteobacteria & Epsilonproteobacteria & Campylobacteraceae & Arcobacter & 0.06 & 0.11 & 1.25 & 0.22 \\
\hline Firmicutes & Bacilli & Enterococcaceae & Enterococcus & 5.93 & 2.55 & 5.19 & 2.88 \\
\hline Firmicutes & Bacilli & Streptococcaceae & Lactococcus & 2.61 & 1.49 & 3.14 & 4.96 \\
\hline Firmicutes & Bacilli & Carnobacteriaceae & Trichococcus & 1.85 & 0.90 & 1.65 & 1.62 \\
\hline Firmicutes & Negativicutes & Acidaminococcaceae & Phascolarctobacterium & 2.17 & 1.79 & 2.14 & 3.31 \\
\hline Firmicutes & Clostridia & Clostridiales_Incertae Sedis XIII & Anaerovorax & 0.46 & 0.83 & 0.72 & 1.05 \\
\hline Bacteroidetes & Bacteroidia & Marinilabiaceae & Alkaliflexus & 5.28 & 3.66 & 4.19 & 4.88 \\
\hline Bacteroidetes & Bacteroidia & Porphyromonadaceae & Bacteroides & 2.32 & 2.69 & 2.50 & 2.63 \\
\hline Bacteroidetes & Bacteroidia & Porphyromonadaceae & Proteiniphilum & 1.68 & 1.66 & 1.47 & 1.31 \\
\hline Actinobacteria & Actinobacteria & Coriobacteriales & Gordonibacter & 9.87 & 7.08 & 9.08 & 6.65 \\
\hline Synergistetes & Synergistia & Synergistaceae & Cloacibacillus & 1.31 & 2.96 & 1.56 & 3.01 \\
\hline Chloroflexi & Anaerolineae & Anaerolineaceae & Levilinea & 0.86 & 0.49 & 0.88 & 1.11 \\
\hline \multirow[t]{2}{*}{ TM7 } & TM7_genera_incertae_sedis & & TM7_genera_incertae_sedis & 1.06 & 1.03 & 0.51 & 0.58 \\
\hline & & & Others & 15.26 & 19.31 & 16.85 & 24.58 \\
\hline
\end{tabular}


four samples fluctuated in a narrow range between 2.79 to $3.98 \%$. Klebsiella is a typical fermentative growth genus and has been proved producing current without a mediator [41-43]. Lactococcus presented function in azo dye decolorization [44], nitro-compound biotransformation [45] and anodic electron transfer [46]. Zhilina et al. revealed that Alkaliflexus was capable of carbohydratefermenting to produce propionate mainly [47].

\subsection{Implications}

Two anodes were preinoculated before assembling in the UBES. Electrochemical active bacteria were selectively enriched by electric field and built the anode biofilms for continuously generating electrons to support cathode reaction. The inoculated anodes were installed into UBES with raw cathodes and likely served as "inoculum" for microbes colonizing on the cathodes. Similar microbial community structures among these electrodes seem to attribute to the "crosstalk" in the same chamber. Biofilms growing on anodes could produce mediators that affect growth and (bio)electrochemical activity of biofilms on other electrodes $[48,49]$.

Microorganisms belonging to Enterobacter, Desulfovibrio and Enterococcus were selected and dominated in all samples under electrochemical condition. These bacteria were reported to be electrochemical active at anode and like implement the anode respiration [32,33]. In addition to transfer electrons to the anode, they also are capable of acquiring electrons from cathode to support metabolism and potential functions. Enterobacter was found to be dominant component in a biocathode microbial community which employed for bioelectrochemically reducing chloramphenicol [10]. Desulfovibrio spp. was reported as a functional species in a microbial electrolysis cell (MEC) equipped with biocathode used for hydrogen production [50]. Microbial community analysis demonstrated that a biocathode was dominated by an Enterococcus species closely related to Enterococcus aquimarinus, which was employed to selectively reduce nitrobenzene to aniline [16]. It is likely that these genera were enriched in the anode biofilms first and then migrated to the cathodes due to the electrodes immersing in the same chamber. The similar microbial community structures among four electrodes and not be obviously altered by neither the polarity nor the position might attribute to these genera presented bidirectional extracellular electron transfer (EET) characteristic.

The biofilms used in BES were usually inoculated with multiple natural sources, such as domestic wastewater, anaerobic and aerobic sludge, river sediment, etc. $[16,19]$. Although the applied electric field could selectively enrich electrochemical active and functional bacteria, there are also some nonfunctional consortia attaching on the electrode and occupying the active site. It may influence the EET, especially in the cathode. Nonfunctional microbes would cover the surface of the cathode and weakened the reduction reaction kinetics.

By introducing microbial catalysis anode in BES, about 1 order of energy consumption decrease was achieved comparing to conventional electrochemical technology. With the microbial catalysis cathode, the overpotential was further decreased and led to further energy savings $[7,16]$. Biocathode normally formed by inoculating enrichment consortium $[10,16]$, but it will take a quite long time and may not enrich microbes that are capable of EET. One feasible strategy to gain an efficient biocathode is enriching electrochemical active microbes in the anode and then inoculating the cathode. Wang et al. removed the ion exchange membrane from a dual chamber BES and led electrochemical active bacteria migrating from the bioanode to the abiotic cathode to build a biocathode for azo dye decolorization [51]. However, ion exchange membrane will substantially increase the operational cost. A recent report revealed an approach to gain efficient biocathode by inversing from an acclimated bioanode [52]. This complicated operation would be a limiting factor to obstruct this technology to application.

To overcome those disadvantages and bring BES closer to practical application, this study proposed a start-up and biofilm establishing strategy that assembling the well startup bioanodes with abiotic cathodes into a single chamber UBES. The functional microbial communities have been identified from both anodes and cathodes. However, the performance of implement this technology under real wastewater condition with fluctuating characteristics, such as non or lower electrons donors, weak buffer system, complex substrates, etc., needs to be further illuminated. Besides, applied voltage in this study was kept at optimized value of $0.5 \mathrm{~V}$ [26] and did not be altered in all experiment. Considering the challenges like electrochemical performance loss towards upscaling [53], the interaction of the applied voltage, electrode material and electrode active microorganisms should be deeply investigated in future research.

\section{Conclusion}

In this work, a single chamber up-flow bioelectrochemical system (UBES) was assembled with two preinoculated anodes and two raw cathodes for azo dye wastewater treatment. Electrode active bacteria could migrate from preinoculated anodes to establish functional biocathodes. Microbial community structures were not obviously altered by neither the polarity nor the position. Microorganisms belonging to Enterobacter, Desulfovibrio and Enterococcus were found to be dominant in all biofilms. This study provides a feasible strategy to build electrode active biofilms in the UBES for azo dye wastewater treatment and gives great inspirations to bring this technology closer to the application.

\section{Acknowledgements}

This research was supported by the National Nature Science Foundation for Distinguished Young Scholars (Grant No. 51225802), the National Nature Science Foundation of China (Grant No. 21577162, 21407164), the China Postdoctoral Science Foundation (Grant No. 2015M580140, No. 2016T90142), the Ministry of Environmental Protection of the People's Republic of China (Major Science and Technology Program for Water Pollution Control and Treatment) (Grant No. 2014ZX07204-005), the "Hundred Talents Program" of the Chinese Academy of Sciences (Grant No.29BR2013001) and Science and Technology Service Network Initiative Project of Chinese Academy of Sciences (Grant No. KFJ-EW-STS-102).

\section{References}

[1] S. You, B. Liu, Y. Gao, Y. Wang, C.Y. Tang, Y. Huang, N. Ren, Monolithic Porous Magnéli-phase Ti407 for Electro-oxidation Treatment of Industrial Wastewater, Electrochim. Acta 214 (2016) 326-335.

[2] C.A. Martínezhuitle, M.A. Rodrigo, I. Sirés, O. Scialdone, Single and Coupled Electrochemical Processes and Reactors for the Abatement of Organic Water Pollutants: A Critical Review, Chem. Rev. 115 (2015).

[3] M.H. Cui, D. Cui, L. Gao, A.J. Wang, H.Y. Cheng, Azo dye decolorization in an upflow bioelectrochemical reactor with domestic wastewater as a cost-effective yet highly efficient electron donor source, Water Res. 105 (2016) 520-526.

[4] M.H. Cui, D. Cui, L. Gao, H.Y. Cheng, A.J. Wang, Efficient azo dye decolorization in a continuous stirred tank reactor (CSTR) with built-in bioelectrochemical system, Bioresour. Technol. 218 (2016) 1307-1311.

[5] M.-H. Cui, D. Cui, B. Liang, T. Sangeetha, A.-J. Wang, H.-Y. Cheng, Decolorization enhancement by optimizing azo dye loading rate in an anaerobic reactor, RSC Adv. 6 (2016) 49995-50001.

[6] D. Cui, Y.-Q. Guo, H.-S. Lee, H.-Y. Cheng, B. Liang, F.-Y. Kong, Y.-Z. Wang, L.-P. Huang, M.-Y. Xu, A.-J. Wang, Efficient azo dye removal in bioelectrochemical system and post-aerobic bioreactor: Optimization and characterization, Chem. Eng. J. 243 (2014) 355-363. 
[7] Y. Mu, R.A. Rozendal, K. Rabaey, J. Keller, Nitrobenzene Removal in Bioelectrochemical Systems, Environ. Sci. Technol. 43 (2009) 8690-8695.

[8] L. Huang, L. Jiang, Q. Wang, X. Quan, J. Yang, L. Chen, Cobalt recovery with simultaneous methane and acetate production in biocathode microbial electrolysis cells, Chem. Eng. J. (2014).

[9] K.V.d.S. Rabaey, Kirsten; Maignien, Lois, Microbial fuel cells for sulfide removal, Environ. Sci. Technol., 40 (2006) 5218-5224.

[10] B. Liang, H.Y. Cheng, D.Y. Kong, S.H. Gao, F. Sun, D. Cui, F.Y. Kong, A.J. Zhou, W.Z Liu, N.Q. Ren, W.M. Wu, A.J. Wang, D.J. Lee, Accelerated reduction of chlorinated nitroaromatic antibiotic chloramphenicol by biocathode, Environ. Sci. Technol. 47 (2013) 5353-5361.

[11] L. Wang, W. Liu, L. Kang, C. Yang, A. Zhou, A. Wang, Enhanced biohydrogen production from waste activated sludge in combined strategy of chemical pretreatment and microbial electrolysis, Int. J. Hydrogen Energy 39 (2014) 11913-11919.

[12] S.A. Cheng, D.F. Xing, D.F. Call, B.E. Logan, Direct Biological Conversion of Electrical Current into Methane by Electromethanogenesis, Environ. Sci. Technol. 43 (2009) 3953-3958.

[13] S. Freguia, K. Rabaey, Z. Yuan, J. Keller, Non-catalyzed cathodic oxygen reduction at graphite granules in microbial fuel cells, Electrochim. Acta 53 (2007) 598-603.

[14] C. Dumas, R. Basseguy, A. Bergel, Electrochemical activity of Geobacter sulfurreducens biofilms on stainless steel anodes, Electrochim. Acta 53 (2008) 5235-5241.

[15] B.E. Logan, K. Rabaey, Conversion of wastes into bioelectricity and chemicals by using microbial electrochemical technologies, Science 337 (2012) 686-690.

[16] A.J. Wang, H.Y. Cheng, B. Liang, N.Q. Ren, D. Cui, N. Lin, B.H. Kim, K. Rabaey, Efficient reduction of nitrobenzene to aniline with a biocatalyzed cathode, Environ. Sci. Technol. 45 (2011) 10186-10193.

[17] M. Rosenbaum, F. Aulenta, M. Villano, L.T. Angenent, Cathodes as electron donors for microbial metabolism: which extracellular electron transfer mechanisms are involved? Bioresour. Technol. 102 (2011) 324-333.

[18] A. Kumar, A. Siggins, K. Katuri, T. Mahony, V. O’Flaherty, P. Lens, D. Leech, Catalytic response of microbial biofilms grown under fixed anode potentials depends on electrochemical cell configuration, Chem. Eng. J. 230 (2013) 532-536.

[19] H. Lin, X. Wu, C. Miller, J. Zhu, Improved performance of microbial fuel cells enriched with natural microbial inocula and treated by electrical current, Biomass Bioenergy 54 (2013) 170-180.

[20] C. Gao, A. Wang, W.M. Wu, Y. Yin, Y.G. Zhao, Enrichment of anodic biofilm inoculated with anaerobic or aerobic sludge in single chambered air-cathode microbial fuel cells, Bioresour. Technol. 167 (2014) 124-132.

[21] C.S. Butler, P. Clauwaert, S.J. Green, W. Verstraete, R. Nerenberg Bioelectrochemical Perchlorate Reduction in a Microbial Fuel Cell, Environ. Sci. Technol. 44 (2010) 4685-4691.

[22] K.B. Gregory, D.R. Lovley, Remediation and recovery of uranium from contaminated subsurface environments with electrodes, Environ. Sci. Technol. 39 (2005) 8943-8947.

[23] D. Cui, Y.Q. Guo, H.S. Lee, W.M. Wu, B. Liang, A.J. Wang, H.Y. Cheng, Enhanced decolorization of azo dye in a small pilot-scale anaerobic baffled reactor coupled with biocatalyzed electrolysis system (ABR-BES): A design suitable for scaling-up, Bioresour. Technol. 163C (2014) 254-261.

[24] M.-H. Cui, D. Cui, H.-S. Lee, B. Liang, A.-J. Wang, H.-Y. Cheng, Effect of electrode position on azo dye removal in an up-flow hybrid anaerobic digestion reactor with built-in bioelectrochemical system, Scientific Reports 6 (2016) 25223.

[25] E.A. Wolin, M.J. Wolin, R.S. Wolfe, Formation of methane by bacterial extracts, J. Biol. Chem 238 (1963) 2882-2886.

[26] D. Cui, Y.Q. Guo, H.Y. Cheng, B. Liang, F.Y. Kong, H.S. Lee, A.J. Wang, Azo dye removal in a membrane-free up-flow biocatalyzed electrolysis reactor coupled with an aerobic bio-contact oxidation reactor, J. Hazard. Mater. 239-240 (2012) 257-264.

[27] X. Jiang, J. Shen, Y. Han, S. Lou, W. Han, X. Sun, J. Li, Y. Mu, L. Wang, Efficient nitro reduction and dechlorination of 2,4-dinitrochlorobenzene through the integration of bioelectrochemical system into upflow anaerobic sludge blanket: A comprehensive study, Water Res. 88 (2016) 257-265.

[28] X.-c. Quan, Y.-p. Quan, K. Tao, Effect of anode aeration on the performance and microbial community of an air-cathode microbial fuel cell, Chem. Eng. J., 210 (2012) 150-156.

[29] K.J. Chae, M.J. Choi, J.W. Lee, K.Y. Kim, I.S. Kim, Effect of different substrates on the performance, bacterial diversity, and bacterial viability in microbial fuel cells, Bioresour. Technol, 100 (2009) 3518-3525.

[30] S. Jung, J.M. Regan, Comparison of anode bacterial communities and performance in microbial fuel cells with different electron donors, Appl. Microbiol. Biotechnol. 77 (2007) 393-402.

[31] A. Moutaouakkil, Y. Zeroual, F.Z. Dzayri, M. Talbi, K. Lee, M. Blaghen, Decolorization of azo dyes with Enterobacter agglomerans immobilized in different supports by using fluidized bed bioreactor, Curr Microbiol 48 (2004) 124-129.

[32] Y. Mohan, S.M.M. Kumar, D. Das, Electricity generation using microbial fuel cells, Int. J. Hydrogen Energy 33 (2008) 423-426.

[33] A.P. Borole, J.R. Mielenz, T.A. Vishnivetskaya, C.Y. Hamilton, Controlling accumulation of fermentation inhibitors in biorefinery recycle water using microbial fuel cells, Biotechnol. Biofuels 2 (2009).

[34] E.S. Yoo, J. Libra, U. Wiesmann, Reduction of azo dyes by desulfovibrio desulfuricans, Water Sci. Technol. 41 (2000) 15-22.

[35] F. Rafii, R. Wynne, T.M. Heinze, D.D. Paine, Mechanism of metronidazoleresistance by isolates of nitroreductase-producing Enterococcus gallinarum and Enterococcus casseliflavus from the human intestinal tract, FEMS Microbiol. Lett. 225 (2003) 195-200.

[36] A. Bafana, T. Chakrabarti, P. Muthal, G. Kanade, Detoxification of benzidinebased azo dye by E. gallinarum: time-course study, Ecotoxicol. Environ. Saf. 72 (2009) 960-964.

[37] W. Handayani, V.I. Meitiniarti, K.H. Timotius, Decolorization of Acid Red 27 and Reactive Red 2 by Enterococcus faecalis under a batch system, World Journal of Microbiology \& Biotechnology 23 (2007) 1239-1244.

[38] Y.K. Wang, G.P. Sheng, W.W. Li, Y.X. Huang, Y.Y. Yu, R.J. Zeng, H.Q. Yu, Development of a novel bioelectrochemical membrane reactor for wastewater treatment, Environ. Sci. Technol, 45 (2011) 9256-9261.

[39] K. Rabaey, N. Boon, S.D. Siciliano, M. Verhaege, W. Verstraete, Biofuel cells select for microbial consortia that self-mediate electron transfer, Appl. Environ. Microbiol. 70 (2004) 5373-5382.

[40] B. Liang, H. Cheng, J.D. Van Nostrand, J. Ma, H. Yu, D. Kong, W. Liu, N. Ren, L. Wu, A. Wang, D.J. Lee, J. Zhou, Microbial community structure and function of Nitrobenzene reduction biocathode in response to carbon source switchover. Water Res. 54 (2014) 137-148.

[41] B.E. Logan, Exoelectrogenic bacteria that power microbial fuel cells, Nat Rev Micro 7 (2009) 375-381.

[42] K.-K. Cheng, Q. Liu, J.-A. Zhang, J.-P. Li, J.-M. Xu, G.-H. Wang, Improved 2,3butanediol production from corncob acid hydrolysate by fed-batch fermentation using Klebsiella oxytoca, Process Biochem. 45 (2010) 613-616.

[43] E. Fernando, T. Keshavarz, G. Kyazze, External resistance as a potential tool for influencing azo dye reductive decolourisation kinetics in microbial fuel cells, Int. Biodeterior. Biodegrad. 89 (2014) 7-14.

[44] S.J. You, J.Y. Teng, Anaerobic decolorization bacteria for the treatment of azo dye in a sequential anaerobic and aerobic membrane bioreactor, J. Taiwan Inst. Chem. Eng. 40 (2009) 500-504.

[45] K.H. Shin, Y. Lim, J.H. Ahn, J. Khil, C.J. Cha, H.G. Hur, Anaerobic biotransformation of dinitrotoluene isomers by Lactococcus lactis subsp lactis strain 27 isolated from earthworm intestine, Chemosphere 61 (2005) 30-39.

[46] M. Masuda, S. Freguia, Y.F. Wang, S. Tsujimura, K. Kano, Flavins contained in yeast extract are exploited for anodic electron transfer by Lactococcus lactis, Bioelectrochemistry 78 (2010) 173-175.

[47] T.N. Zhilina, R. Appel, C. Probian, E.L. Brossa, J. Harder, F. Widdel, G.A. Zavarzin, Alkaliflexus imshenetskii gen. nov sp nov., a new alkaliphilic gliding carbohydrate-fermenting bacterium with propionate formation from a soda lake, Arch. Microbiol. 182 (2004) 244-253.

[48] X. Zhu, M.D. Yates, M.C. Hatzell, H. Ananda Rao, P.E. Saikaly, B.E. Logan, Microbial Community Composition Is Unaffected by Anode Potential, Environ. Sci. Technol. (2014).

[49] C.I. Torres, R. Krajmalnik-Brown, P. Parameswaran, A.K. Marcus, G. Wanger, Y.A. Gorby, B.E. Rittmann, Selecting anode-respiring bacteria based on anode potential: phylogenetic, electrochemical, and microscopic characterization, Environ. Sci. Technol, 43 (2009) 9519-9524.

[50] E. Croese, M.A. Pereira, G.J. Euverink, A.J. Stams, J.S. Geelhoed, Analysis of the microbial community of the biocathode of a hydrogen-producing microbial electrolysis cell, Appl. Microbiol. Biotechnol. 92 (2011) 1083-1093.

[51] Y.Z. Wang, A.J. Wang, W.Z. Liu, D.Y. Kong, W.B. Tan, C. Liu, Accelerated azo dye removal by biocathode formation in single-chamber biocatalyzed electrolysis systems, Bioresour. Technol. 146 (2013) 740-743.

[52] D.Y. Kong, B. Liang, H. Yun, A.J. Wang, N.Q. Ren, Bioanode and Inversion of Bioanode to Biocathode for the Degradation of Antibiotic Chloramphenicol, Environ. Sci. 36 (2015) 1352-1358.

[53] S.K. Butti, G. Velvizhi, M.L.K. Sulonen, J.M. Haavisto, E. Oguz Koroglu, A. Yusuf Cetinkaya, S. Singh, D. Arya, J. Annie Modestra, K. Vamsi Krishna, A. Verma, B. Ozkaya, A.-M. Lakaniemi, J.A. Puhakka, S. Venkata Mohan, Microbial electrochemical technologies with the perspective of harnessing bioenergy: Maneuvering towards upscaling, Renewable Sustainable Energy Rev. 53 (2016) 462-476. 